\title{
The Diagnostic Accuracy Of Procalcitonin for Urinary Tract Infection in Hospitalized Older Adults: a Prospective Study
}

\author{
Justin J. Choi, MD ${ }^{1,2} \mathbb{D}$, Matthew W. McCarthy, MD², Kerry K. Meltzer, MD², \\ Anna Cornelius-Schecter, BA ${ }^{3}$, Assem Jabri, $M D^{7}$, Evgeniya Reshetnyak, $P h D^{7}$, \\ Samprit Banerjee, $P h D^{4}$, Lars F. Westblade, PhD ${ }^{5,6}$, Saurabh Mehta, MBBS7, \\ Matthew S. Simon, MD ${ }^{2,6}$, Zhen Zhao, $P h D^{5}$, and Marshall J. Glesby, MD2,4,6
}

\begin{abstract}
'Division of General Internal Medicine, Department of Medicine, Weill Cornell Medicine, 420 East 70th Street, LH-355, New York, NY 10021, USA; ${ }^{2}$ NewYork-Presbyterian Hospital/Weill Cornell Medical Center, New York, NY, USA; ${ }^{3}$ MD Program, Weill Cornell Medicine, New York, NY, USA; ${ }^{4}$ Department of Population Health Sciences, Weill Cornell Medicine, New York, NY, USA; ${ }^{5}$ Department of Pathology and Laboratory Medicine, Weill Cornell Medicine, New York, NY, USA; ${ }^{6}$ Division of Infectious Diseases, Department of Medicine, Weill Cornell Medicine, New York, NY, USA; ${ }^{7}$ Institute for Nutritional Sciences, Global Health, and Technology, Division of Nutritional Sciences, College of Human Ecology, Cornell University, Ithaca, NY, USA
\end{abstract}

Background: The diagnosis of urinary tract infection (UTI) is challenging among hospitalized older adults, particularly among those with altered mental status.

Objective: To determine the diagnostic accuracy of procalcitonin (PCT) for UTI in hospitalized older adults.

Design: We performed a prospective cohort study of older adults ( $\geq 65$ years old) admitted to a single hospital with evidence of pyuria on urinalysis. PCT was tested on initial blood samples. The reference standard was a clinical definition that included the presence of a positive urine culture and any symptom or sign of infection referable to the genitourinary tract. We also surveyed the treating physicians for their clinical judgment and performed expert adjudication of cases for the determination of UTI.

Participants: Two hundred twenty-nine study participants at a major academic medical center.

Main Measures: We calculated the area under the receiver operating characteristic curve (AUC) of PCT for the diagnosis of UTI.

Key Results: In this study cohort, 61 (27\%) participants met clinical criteria for UTI. The median age of the overall cohort was 82.6 (IQR 74.9-89.7) years. The AUC of PCT for the diagnosis of UTI was 0.56 (95\% CI, 0.46-0.65). A series of sensitivity analyses on UTI definition, which included using a decreased threshold for bacteriuria, the treating physicians' clinical judgment, and independent infectious disease specialist adjudication, confirmed the negative result.

Conclusions: Our findings demonstrate that PCT has limited value in the diagnosis of UTI among hospitalized older adults. Clinicians should be cautious using PCT for the diagnosis of UTI in hospitalized older adults.

KEY WORDS: procalcitonin; diagnostic accuracy; urinary tract infection; older adults

J Gen Intern Med 37(14):3663-9

DOI: $10.1007 / \mathrm{s} 11606-021-07265-8$

(c) Society of General Internal Medicine 2021

Received August 13, 2021

Accepted October 29, 2021

Published online January 8, 2022

\section{INTRODUCTION}

Urinary tract infections (UTIs) are the second leading infectious cause of hospitalization and the leading infectious cause of emergency department visits among older adults in the USA. ${ }^{1,2}$ The diagnosis of UTI is challenging in older adults and controversial among clinicians. ${ }^{3,4}$ UTI is typically a clinical diagnosis made on the presence of urinary symptoms and may be supported by the presence of pyuria and/or bacteriuria. However, eliciting urinary symptoms in hospitalized older adults may be limited by cognitive impairment or altered mental status. ${ }^{5}$

Further complicating the diagnostic evaluation of UTI is the turnaround time of urine culture results, which can be days after a decision must be made on whether to initiate or withhold antibiotics. In addition, there is a high prevalence of asymptomatic bacteriuria in older adults. ${ }^{6-8}$ Antibiotic therapy for asymptomatic bacteriuria in older adults is not recommended by current guidelines. ${ }^{9-11}$ Inappropriate inpatient antibiotic use is often attributable to misdiagnosis. ${ }^{12,13}$

Procalcitonin (PCT) is a biomarker used to identify acute bacterial infections and has been incorporated in clinical algorithms to safely reduce antibiotic use. ${ }^{14-16}$ One randomized clinical trial implemented an algorithm based on clinical symptoms, PCT, and pyuria for the management of UTI in the emergency department and resulted in a $30 \%$ reduction in antibiotic use compared to usual care. ${ }^{17}$ Other studies have suggested that PCT is helpful in the diagnosis and management of UTI; however, these studies were mostly performed retrospectively, in the outpatient setting, or in pediatric populations. ${ }^{18-23}$ A recent crosssectional study of nursing home residents showed that point-of-care PCT was not suitable for diagnosing UTI. ${ }^{24}$

The aim of this study was to determine the diagnostic accuracy of PCT for UTI among hospitalized older adults with clinical suspicion for UTI. In addition, since urine culture positivity is highly influential in the diagnosis and management of UTIs in the inpatient setting, we evaluated the common clinical variables on admission that predict a positive urine culture. 


\section{METHODS}

\section{Study Design}

We performed a prospective observational cohort study at NewYork-Presbyterian/Weill Cornell Medical Center (NYP/WCMC), an 862-bed major academic medical center located in New York City, from August 2019 to January 2021. Study enrollment was interrupted from March to July 2020 due to the COVID-19 pandemic. We used a convenience sampling strategy and screened eligible patients during weekdays using an electronic health record list of new general medicine patients admitted in the previous $24 \mathrm{~h}$. Informed consent was obtained from either the patient or their legally authorized representative. This study was approved by the Weill Cornell Medicine Institutional Review Board.

\section{Study Population}

Eligible study participants included older adults ( $\geq 65$ years old) who were admitted to a general medicine service (i.e., non-critically ill patients) and had evidence of pyuria ( $\geq 10 \mathrm{wbc} / \mathrm{hpf}$ ) on urinalysis performed on a highquality specimen $(<10$ epithelial cells/hpf) as part of an evaluation for non-catheter-related UTI. The exclusion criteria were as follows: (1) evidence on admission of an infectious process not referable to the genitourinary tract, (2) an estimated glomerular filtration rate $<30 \mathrm{~mL} / \mathrm{min}$, (3) recent major trauma or surgery within 1 month, (4) recent antibiotic use within 1 week, and (5) inability to obtain informed consent from the participant or legally authorized representative within $72 \mathrm{~h}$ of admission.

\section{Index Test}

Serum PCT was analyzed in the NYP/WCMC clinical laboratory using the Food and Drug Administration (FDA)-approved Roche Cobas e411 analyzer for the Elecsys BRAHMS PCT assay [Roche Diagnostics, Indianapolis, IN]. The reportable measurement range is 0.01 to $400.00 \mathrm{ng} / \mathrm{mL}$. PCT measurements were performed on remnant blood samples that were collected as part of routine clinical care in the emergency department. PCT results were collected for research purposes only and were not made available to the clinicians, data collectors, or data analysts who adjudicated outcomes. We did not prevent clinicians from ordering PCT as part of routine care.

\section{Reference Standard}

While no consensus definition of UTI exists, we used the following definition based on clinical criteria derived from the literature and our clinical experience $e^{4,8,25,26}$ : (1) pyuria with $\geq 10 \mathrm{wbc} / \mathrm{hpf}$ on urinalysis (all patients met this criterion by default given the study inclusion criteria), (2) bacteriuria with $\geq 10^{5}$ colony-forming units (CFUs)/mL and no more than 2 bacterial species recovered in urine culture, and (3) any of the following symptoms or signs: temperature $\geq 38^{\circ} \mathrm{C}$, urinary frequency or urgency, dysuria, suprapubic tenderness, costovertebral angle (i.e., flank) pain or tenderness not explained by other diagnoses, or radiographic evidence of cystitis or pyelonephritis on ultrasound on computed tomography imaging. Study investigators were blinded to the index test when adjudicating for UTI through a detailed chart review.

\section{Data Collection}

Demographic information and clinical variables present on hospital admission such as vital signs (temperature, heart rate, blood pressure, respiratory rate) and laboratory data (complete blood count and comprehensive metabolic panel) were collected using REDCap electronic data capture tools..$^{27,28}$ Detailed chart reviews of electronic health record documentation were performed manually by trained research assistants with clinical experience to collect clinical information such as co-morbid conditions, symptoms, physical examination signs, microbiological testing results, and radiology reports.

\section{Statistical Analysis}

We used descriptive statistics as appropriate such as median and interquartile range (IQR) for all continuous variables and proportions for categorical variables. Comparisons between patients with and without UTI were performed using MannWhitney tests, chi-squared tests, or Fisher's exact tests, as appropriate. We calculated receiver operating characteristic (ROC) curves, their area under the curve (AUC), and 95\% confidence interval (CI). We derived an empirical cutpoint for PCT using the Youden index method, ${ }^{29}$ then calculated its sensitivity and specificity. A subgroup analysis was performed based on sex. There was neither missing data nor indeterminate results for the index test. Missing reference standard results in clinical documentation for presenting symptoms and signs were assumed to be absent, while cases with missing urine culture results were excluded from the primary analysis.

We also performed multivariate analysis using Poisson regression to predict a positive urine culture (defined as bacteriuria with $\geq 10^{5} \mathrm{CFUs} / \mathrm{mL}$ ) that included the following independent variables: age, sex, urinary frequency, or urgency, dysuria, fever, suprapubic tenderness, costovertebral angle tenderness, altered mental status, severe pyuria (defined as $>50 \mathrm{wbc} / \mathrm{hpf}$ ), and peripheral white blood cell count. We calculated the relative risk (RR) and 95\% CIs to determine the measures of association. 


\section{Sensitivity Analyses}

Given the absence of a true gold standard, ${ }^{8,30}$ we performed a series of sensitivity analyses to further evaluate the diagnostic accuracy of PCT based on different definitions for UTI. First, we lowered the threshold for bacteriuria in the reference standard from $\geq 10^{5}$ to $\geq 10^{4} \mathrm{CFUs} / \mathrm{mL}$. ${ }^{31}$ Second, we surveyed attending physicians directing the care of the study participants for their judgment on the presence of UTI, with response choices of no, possibly, probably, and yes (i.e., definitely). Surveys were sent 5 days after patient enrollment in the study. Finally, infectious diseases specialists (MWM or MSS) retrospectively adjudicated cases to determine the presence of UTI while blinded to the index test and reference standard used.

\section{Sample Size Calculation}

We performed a sample size calculation using previously published methods that determine sample sizes that can statistically detect differences in the accuracy of diagnostic techniques based on ROC curve analysis using estimated prevalence and expected AUC. ${ }^{32,33}$ To our knowledge, no study has reported the prevalence of UTI in older adults hospitalized with evidence of pyuria. Based on an AUC of 0.87 for urine dipstick, which is currently the best available non-invasive marker for the rapid diagnosis of urinary tract infection, our study aimed to find a highly accurate test with an AUC above $0.87 .{ }^{34}$ We estimated the prevalence of UTI in our study population to be $60 \%$ based on our experience. A total of 217 subjects would produce a sample AUC of 0.85 and a two-sided $95 \%$ CI with a width of 0.10 when the prevalence is $60 \%$. Estimating a $5 \%$ dropout rate and/or to account for potential missing key data for the aforementioned sample, we planned to enroll 228 study participants.

\section{RESULTS}

\section{Baseline Characteristics}

A total of 229 study participants were included in the study (see Flow Diagram in Supplementary Figure). Table 1 shows the baseline characteristics of study participants. The median age of the overall cohort was 82.6 years (IQR 74.9-89.7), 64\% were women, 54\% White, $11 \%$ Hispanic, 9\% Black, and 7\% Asian. The UTI subgroup defined by the reference standard based on clinical criteria had 61 (27\%) participants, while the "no UTI" subgroup had 158 (69\%) participants. Ten participants were excluded from the UTI and "no UTI" subgroups due to the absence of urine culture collection.

\section{Clinical Characteristics}

Table 2 shows the clinical characteristics upon hospital admission, including presenting urinary symptoms, physical examination findings, and laboratory findings. Sixteen (7\%) participants were found to have bacteremia - in all 16 cases, the isolated bacterial pathogen in blood cultures matched the same pathogen identified in urine cultures. Sixty-six (29\%) participants had computed tomography imaging and $46(20 \%)$ had ultrasound imaging of the abdomen and/or pelvis - 30 had evidence of pyelonephritis and 11 had evidence of cystitis.

\section{Diagnostic Accuracy of Procalcitonin}

The serum concentrations of PCT in the overall study cohort were relatively low; the median value was $0.08 \mathrm{ng} / \mathrm{mL}$ (IQR $0.05-0.19$ ) with a minimum value of $0.01 \mathrm{ng} / \mathrm{mL}$ and maximum value of $30.46 \mathrm{ng} / \mathrm{mL}$. Figure 1 shows the ROC curve analysis for PCT to detect UTI as defined by clinical criteria. PCT performed poorly in its diagnostic performance with an AUC of 0.56 (95\% CI, 0.46-0.65). Results were similar when stratified by sex, with an AUC of 0.47 (95\% CI, $0.35-0.59)$ among females and an AUC of 0.58 (95\% CI $0.43-0.73$ ) among males.

The empirical estimated cutpoint for PCT was $0.18 \mathrm{ng} / \mathrm{mL}$, which resulted in a sensitivity of 38\% (95\% CI, 26-52\%), specificity of $79 \%$ (95\% CI, 71-85\%), positive predictive value of $40 \%$ (95\% CI, 27-53\%), and negative predictive value of $76 \%$ (95\% CI, 69-83\%). In a post hoc exploratory analysis, we classified cases of UTI into lower UTI $(n=25)$ and upper UTI $(n=36)$ based on definitions provided in the Supplementary Materials. For lower UTI, the sensitivity, specificity, positive predictive value, and negative predictive value at previously defined PCT cutpoint $<0.18 \mathrm{ng} / \mathrm{mL}$ were $24 \%$ (95\% CI, 9-45\%), 78\% (95\% CI, 71-84\%), $15 \%$ (95\% CI, 6-29\%), and 87\% (95\% CI, 80-92\%), respectively. For upper UTI, the sensitivity, specificity, positive predictive value, and negative predictive value of PCT $<0.18 \mathrm{ng} / \mathrm{mL}$ were $47 \%$ (95\% CI, 30-65\%), 78\% (95\% CI, 71-83\%), 29\% (95\% CI, 18-43\%), and 88\% (95\% CI, 82-93\%), respectively.

\section{Sensitivity Analyses}

When the threshold of bacteriuria in the reference standard was reduced from $\geq 10^{5}$ to $\geq 10^{4} \mathrm{CFUs} / \mathrm{mL}, 69$ participants met clinical criteria for UTI and 150 participants did not. PCT was unable to discriminate between UTI and "no UTI" under this revised definition with an AUC of 0.56 (95\% CI $0.48-0.65)$.

In our survey of the attending physicians in charge of the care of study participants, the response rate was $89 \%$ $(n=204)$. Overall, $40 \%$ of cases were judged by the attending physician as "definitely" having a UTI, and $32 \%$ of cases 
Table 1 Baseline Characteristics for the Study Cohort and Comparison Between Patients With and Without Urinary Tract Infection
Abbreviations: $I Q R$, interquartile range; $U T I$, urinary tract infection

${ }^{a}$ Active cancer, defined by any of the following: diagnosed or receiving therapy within 6 months of presentation; recurrent or metastatic cancer.

\begin{tabular}{|c|c|c|c|c|}
\hline \multirow[t]{2}{*}{ Characteristic } & \multicolumn{4}{|l|}{$n(\%)$} \\
\hline & Overall $(n=229)$ & UTI $(n=61)$ & No UTI $(n=158)$ & $P$ value \\
\hline \multicolumn{5}{|l|}{ Urinary symptoms } \\
\hline Dysuria & $28(12)$ & $19(31)$ & $9(6)$ & $<0.001$ \\
\hline Urinary frequency or urgency & $30(13)$ & $24(39)$ & $6(4)$ & $<0.001$ \\
\hline Urinary retention & $21(9)$ & $7(12)$ & $14(9)$ & 0.56 \\
\hline \multicolumn{5}{|l|}{ Physical examination findings } \\
\hline Fever $^{\mathrm{a}}$ & $29(13)$ & $20(33)$ & $9(6)$ & $<0.001$ \\
\hline Tachycardia $^{\mathrm{b}}$ & $125(55)$ & $37(61)$ & $83(53)$ & 0.28 \\
\hline Tachypnea $^{c}$ & $47(21)$ & $14(23)$ & $32(20)$ & 0.66 \\
\hline Altered mental status & $67(29)$ & $17(28)$ & $50(32)$ & 0.59 \\
\hline Flank tenderness & $15(7)$ & $9(15)$ & $5(3)$ & 0.002 \\
\hline Suprapubic tenderness & $21(9)$ & $11(18)$ & $10(6)$ & 0.01 \\
\hline \multicolumn{5}{|l|}{ Laboratory findings } \\
\hline Bacteriuria $\left(>10^{5} \mathrm{CFU} / \mathrm{mL}\right)$ & $126(55)$ & $61(100)$ & $64(41)$ & $<0.001$ \\
\hline White blood cell count, median (IQR) & $9.2(6.6,12.0)$ & $10.0(7.2,13.9)$ & $9.0(6.5,11.1)$ & 0.07 \\
\hline
\end{tabular}

Abbreviations: $C F U$, colony-forming units; $I Q R$, interquartile range; $U T I$, urinary tract infection

${ }^{\text {a}}$ Defined as temperature $\geq 38^{\circ} \mathrm{C}$

${ }^{\mathrm{b}}$ Defined as $>90$ beats per minute

${ }^{c}$ Defined as $>20$ breaths per minute

\begin{tabular}{|c|c|c|c|c|}
\hline \multirow[t]{2}{*}{ Characteristic } & \multicolumn{4}{|l|}{$n(\%)$} \\
\hline & Overall $(n=229)$ & UTI $(n=61)$ & No UTI $(n=158)$ & $P$ value \\
\hline Age, median (IQR), years & $82.6(74.9-89.7)$ & $81.1(74.4-87.1)$ & $83.6(75.4-90.4)$ & 0.057 \\
\hline Female & $146(64)$ & $36(59)$ & $104(66)$ & 0.35 \\
\hline Race & & & & 0.20 \\
\hline White & $124(54)$ & $34(56)$ & $84(53)$ & \\
\hline Black & $20(9)$ & $3(5)$ & $15(10)$ & \\
\hline Asian & $16(7)$ & $1(2)$ & $14(9)$ & \\
\hline Pacific Islander & $2(1)$ & $1(2)$ & $1(1)$ & \\
\hline American Indian or Alaska Native & $1(<1)$ & $1(2)$ & $0(0)$ & \\
\hline Other race & $58(25)$ & $19(31)$ & $38(24)$ & \\
\hline Unknown & $8(4)$ & $2(3)$ & $6(4)$ & \\
\hline Ethnicity & & & & 0.19 \\
\hline Hispanic or Latino & $24(11)$ & $10(16)$ & $14(9)$ & \\
\hline Not Hispanic or Latino & $183(80)$ & 47 (77) & $126(80)$ & \\
\hline Unknown & $22(10)$ & $4(7)$ & $18(11)$ & \\
\hline Place of residence & & & & 0.72 \\
\hline Home (independent living) & $119(52)$ & $29(48)$ & $82(52)$ & \\
\hline Home with home care services & $86(38)$ & $26(43)$ & $58(37)$ & \\
\hline Skilled nursing facility & $22(10)$ & $5(8)$ & $17(11)$ & \\
\hline Acute care hospital & $2(1)$ & $1(2)$ & $1(1)$ & \\
\hline \multicolumn{5}{|l|}{ Comorbidities } \\
\hline Active cancer ${ }^{\mathrm{a}}$ & $46(20)$ & $16(26)$ & $27(17)$ & 0.13 \\
\hline Cognitive impairment & $55(24)$ & $12(20)$ & $39(25)$ & 0.43 \\
\hline Coronary artery disease & $70(31)$ & $22(36)$ & $44(28)$ & 0.23 \\
\hline Dementia & $57(25)$ & $11(18)$ & $43(27)$ & 0.16 \\
\hline Diabetes mellitus & $62(27)$ & $21(34)$ & $38(24)$ & 0.12 \\
\hline
\end{tabular}

able 2 Clinica

Coracteristics for the Study

Between Patients With and Without Urinary Tract Infection 
Table 3 Poisson Regression Analysis of Clinical Variables Predicting a Positive Urine Culture

\begin{tabular}{lll}
\hline Clinical variable & Relative risk (95\% CI) & $P$ value \\
\hline Age & $1.00(0.98-1.01)$ & 0.64 \\
Sex & $1.08(0.85-1.36)$ & 0.53 \\
Urinary frequency or urgency & $1.50(1.15-1.96)$ & 0.003 \\
Suprapubic tenderness & $0.79(0.52-1.18)$ & 0.25 \\
Costovertebral angle tenderness & $1.06(0.71-1.59)$ & 0.78 \\
Altered mental status & $1.27(0.997-1.62)$ & 0.053 \\
Fever & $1.14(0.85-1.54)$ & 0.38 \\
Severe pyuria (>50 wbc/hpf) & $1.37(1.08-1.73)$ & 0.01 \\
Peripheral white blood cell count & $0.98(0.96-1.01)$ & 0.24 \\
\hline
\end{tabular}

Note: A positive urine culture was defined as bacteriuria with $\geq 10^{5}$ CFUs/mL

were judged to be either "possibly" or "probably" UTI (Supplementary Table). PCT was unable to discriminate between "no UTI" and a composite of "possibly, probably, and definitely UTI" as determined by physician judgment with an AUC of 0.50 (95\% CI, 0.41-0.59). Similarly, in our expert adjudication of 160 cases for the presence of UTI, PCT had an AUC of 0.50 (95\% CI, 0.41-0.60).

\section{Prediction of Positive Urine Culture}

Table 3 shows a multivariate analysis using a Poisson regression to predict a positive urine culture (defined as bacteriuria with $\geq 10^{5} \mathrm{CFUs} / \mathrm{mL}$ ). Among the variables included in the

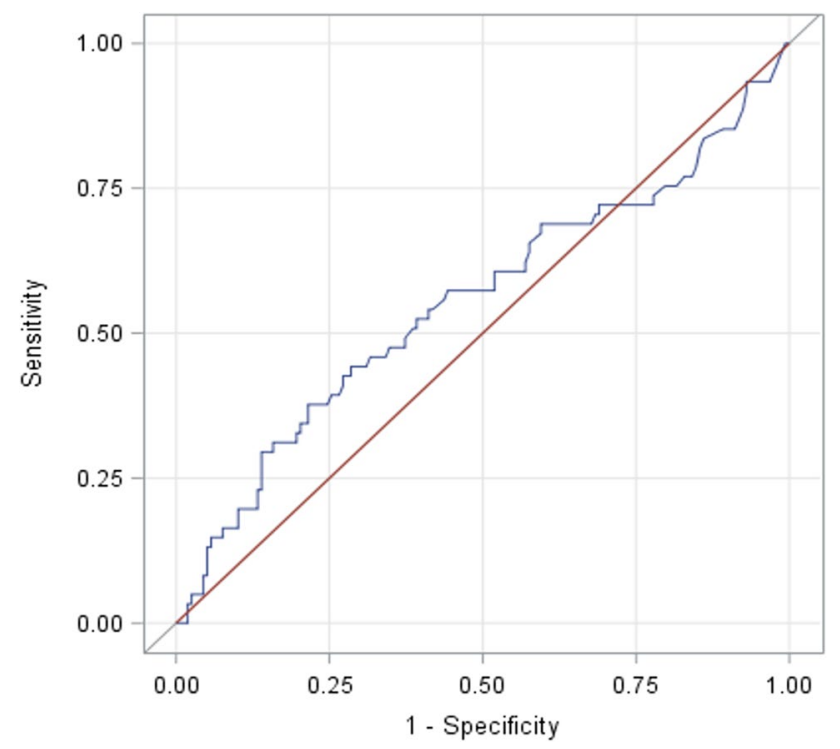

Figure 1. Primary ROC curve analysis for PCT to detect the presence of UTI. Receiver operating characteristic (ROC) curve for procalcitonin (PCT) to detect urinary tract infection (UTI) defined by clinical criteria. The area under the curve is $0.56(95 \%$ CI, 0.46-0.65; $P=0.24)$. model, the presence of urinary frequency or urgency (RR 1.50; 95\% CI, 1.15-1.96) and severe pyuria (RR 1.37; 95\% CI, 1.08-1.73) demonstrated statistically significant associations with a positive urine culture. The presence of altered mental status did not reach statistical significance (RR 1.27; 95\% CI, 0.997-1.62).

\section{DISCUSSION}

This prospective study of hospitalized older adults with initial clinical suspicion for non-catheter-related UTI demonstrated poor diagnostic performance of PCT for the diagnosis of UTI, whether defined by clinical criteria, physician judgment, or expert adjudication by infectious diseases specialists. This negative result is in contrast with prior studies of PCT for the diagnosis and management of UTI in adults.

A single-center retrospective study by Levine et al. analyzed 293 adults who presented to the emergency department and underwent both UTI evaluation and PCT testing found that a PCT cutpoint of $<0.25 \mathrm{ng} / \mathrm{mL}$ had a strong negative predictive value of $91 \%$ for ruling out UTI. ${ }^{19}$ In comparison with our study, a more stringent empirical cutpoint of $<0.18 \mathrm{ng} / \mathrm{mL}$ had lower sensitivity (38\%) and specificity (78\%) and yielded a negative predictive value of only $77 \%$. Potential study factors that could explain this discrepancy in diagnostic performance include the retrospective design used by Levine et al. and their exclusion of 1800 patients among 2093 records that were reviewed due to the absence of PCT testing, exclusion of patients with symptoms or signs of pyelonephritis (e.g., flank pain, fever), and exclusion of immunocompromised patients.

Another retrospective study by Rothe et al. examined cases of 183 hospitalized patients with systemic urinary infection and bacteriuria, using a more lenient threshold of $\geq 10^{3} \mathrm{CFUs} / \mathrm{mL}$ in both catheterized and non-catheterized patients, and excluded patients with negative urine cultures. ${ }^{35}$ Rothe et al. examined the diagnostic accuracy of PCT to detect true bacteremia and found a negative predictive value of $89 \%$ using a PCT cutpoint of $<0.25 \mathrm{ng} / \mathrm{mL}$. However, $26 \%$ of cases in this analysis included bacterial infections not referrable to the genitourinary tract (pneumonia, $n=30$; venous access infection, $n=9$; skin and soft tissue infection, $n=9$ ). While our study only detected $7 \%$ of UTI cases with bacteremia, our analysis in upper UTI found poor diagnostic performance of PCT in identifying symptoms and signs suggestive of systemic urinary infection and bacteriuria.

Drozdov et al. conducted a small randomized controlled trial of 125 patients with a primary diagnosis of community-acquired non-catheter-related UTI at a single hospital in Switzerland found that a PCT-pyuria-based algorithm with serial assessments every 2 days while on therapy safely reduced antibiotic exposure by $30 \%$ compared with usual care. ${ }^{17}$ The study population in this trial was relatively 
younger, included a higher proportion of women, and included both hospitalized patients as well as patients discharged from the emergency department. This study did not evaluate the diagnostic accuracy of PCT.

Our findings add to the literature that PCT is not a reliable biomarker for the diagnosis of UTI among hospitalized older adults and should not be used by clinicians to determine the likelihood of UTI to make initial antibiotic decisions (i.e., whether to initiate or withhold antibiotics). Our negative result corroborates findings in a recent study of nursing home residents, which showed that point-of-care PCT is not reliable for distinguishing UTI and asymptomatic bacteriuria. ${ }^{24}$ It is still possible that there is a role for PCT to inform decisions around early discontinuation of antibiotics being prescribed for UTI, whether confirmed or not, which is consistent with the recommended approach when using PCT in other settings. In addition, a post hoc exploratory analysis in our study found that PCT $<0.18 \mathrm{ng} / \mathrm{mL}$ had a negative predictive value of $88 \%$ for diagnosis of an upper UTI. However, further investigation in the form of prospective studies or randomized clinical trials is needed to address the following: (a) the role of PCT in early discontinuation of antibiotics for UTI and (b) its role in distinguishing lower and upper UTI syndromes among hospitalized adults.

In current practice, the results of the urine culture are considered a key factor in deciding antibiotic therapy for hospitalized older adults with clinical suspicion for UTI. For example, antibiotics may be discontinued when the urine culture shows no growth of any pathogen, or they may be continued to complete a full course of antibiotic therapy if it shows substantial bacteriuria. The limitation in clinical practice is that urine culture results are not available until days after the initial evaluation for antibiotic decisions for UTI. We found that the best predictors of a positive urine culture (bacteriuria $\geq 10^{5} \mathrm{CFUs} / \mathrm{mL}$ ) were the presence of urinary frequency or urgency and a marked elevation of urinary leukocytes with $>50 \mathrm{wbc} / \mathrm{hpf}$. These results are consistent with findings reported in the largest systematic review to date determining the utility of history, physical, and urinalysis findings in predicting a positive urine culture with bacteriuria $\geq 10^{5} \mathrm{CFUs} / \mathrm{mL}$ among women who present to the emergency department with suspected $\mathrm{UTI}^{36}$; however, the included studies did not evaluate the predictive ability of altered mental status for positive urine cultures. In our study, the presence of altered mental status did not reach statistical significance in its association with a positive urine culture. It remains controversial how clinicians can attribute altered mental status to a true UTI versus asymptomatic bacteriuria among hospitalized older adults.

The major strength of this study is the prospective evaluation of PCT as a diagnostic biomarker in a moderate-sized sample of hospitalized older adults. Another strength is that both clinicians and outcome adjudicators were blinded to PCT results. This study also has several limitations. First, a major limitation is the lack of a gold standard. It is possible that both over-diagnosis and under-diagnosis of UTI in our study occurred, even in patients with intact mental status. Second, this is a highly selected sample from a single center as a relatively high proportion of eligible patients had to be excluded for a variety of reasons. Third, there is incorporation bias given that the reference standard for urinary tract infection includes the presence of pyuria on urinalysis, which was part of the study inclusion criteria. In addition, there were 13 occurrences of PCT testing as part of clinical care, which were available to the attending physicians and outcome adjudicators. Incorporation bias may overestimate both sensitivity and specificity of the index test. Fourth, over $70 \%$ of patients did not undergo imaging to evaluate for radiographic evidence of a urinary tract infection, introducing a partial verification bias to our study. This may overestimate the sensitivity of the index test given the potential underdetection of false negatives. Given the overwhelming negative result of our study, we believe that the incorporation bias and partial verification bias were ultimately not consequential for the interpretation of the study results.

In conclusion, our prospective study reveals that PCT is a poor diagnostic marker for identifying UTI in hospitalized older adults. Clinicians should be cautious using PCT for the diagnosis of UTI in hospitalized older adults.

Supplementary Information The online version contains supplementary material available at https://doi.org/10. 1007/s11606-021-07265-8.

Acknowledgements: We would like to acknowledge Shoran Tamura, Noah Rossen, Joanna Quintero, Oscar Vargas, and Mangala Rajan for their statistical, technical, and administrative assistance in the conduct of this study. We also thank Drs. Olaf Andersen, Alvin Mushlin, and Michael Satlin for their critical review of earlier drafts of this manuscript. Finally, we want to express deep gratitude to the patients who enrolled in this study and the many dedicated clinicians who cared for them and supported this research.

Corresponding Author: Justin J. Choi, MD; Division of General Internal Medicine, Department of Medicine, Weill Cornell Medicine, 420 East 70th Street, LH-355, New York, NY 10021, USA (e-mail: juc9107@med.cornell.edu).

Funding This work was supported by NewYork-Presbyterian Hospital (NYPH) and Weill Cornell Medical College (WCMC), including the Clinical and Translational Science Center (CTSC) (UL1 TR000457), the National Institutes of Health/National Center for Advancing Translational Sciences (KL2-TR-002385) to J.J.C, and an Investigator-Initiated Study award granted by Roche Diagnostics. Roche Diagnostics had no role in the study design, data analysis, or writing of this manuscript. 
Data Availability The datasets during and/or analyzed during the current study are available from the corresponding author on reasonable request.

\section{Declarations:}

Conflict of Interest: J.J.C. has received research support and consulting fees from Roche Diagnostics and Allergan. M.J.G. has received research support from Gilead Sciences and Regeneron, has served as a consultant to Enzychem, ReAlta Life Sciences, Regeneron, and Sobi, and has received royalties from Springer and UpToDate. L.F.W. has received consulting fees from Roche Molecular Systems, Inc., Shionogi, Inc., and Talis Biomedical, and research support from Accelerate Diagnostics, Inc., BioFire Diagnostics, LLC, and Roche Molecular Systems, Inc.

\section{REFERENCES}

1. Curns AT, Holman RC, Sejvar JJ, Owings MF, Schonberger LB. Infectious disease hospitalizations among older adults in the United States from 1990 through 2002. Arch Intern Med 2005; 165:2514-2520.

2. Goto I, Yoshida K, Tsugawa Y, Camargo CA, Hasegawa K. Infectious Disease-Related Emergency Department Visits of Elderly Adults in the United States, 2011-2012. J Am Geriatr Soc 2016; 64:31-36.

3. Froom $\mathbf{P}$, Shimoni $\mathbf{Z}$. The uncertainties of the diagnosis and treatment of a suspected urinary tract infection in elderly hospitalized patients. Expert Rev Anti-Infect Ther 2018; 16:763-770.

4. Woodford HJ, George J. Diagnosis and management of urinary tract infection in hospitalized older people. J Am Geriatr Soc 2009; 57:107-114.

5. Detweiler K, Mayers D, Fletcher SG. Bacteruria and urinary tract infections in the elderly. Urol Clin North Am 2015; 42:561-568.

6. Nicolle LE. Asymptomatic bacteriuria in the elderly. Infect Dis Clin N Am 1997; 11:647-662.

7. Monane M, Gurwitz JH, Lipsitz LA, Glynn RJ, Choodnovskiy I, Avorn J. Epidemiologic and diagnostic aspects of bacteriuria: a longitudinal study in older women. J Am Geriatr Soc 1995; 43:618-622.

8. Rowe TA, Juthani-Mehta M. Urinary tract infection in older adults. Aging Health 2013; 9.

9. Nicolle LE, Bradley S, Colgan R, et al. Infectious Diseases Society of America guidelines for the diagnosis and treatment of asymptomatic bacteriuria in adults. Clin Infect Dis 2005; 40:643-654.

10. McKenzie R, Stewart MT, Bellantoni MF, Finucane TE. Bacteriuria in individuals who become delirious. Am J Med 2014; 127:255-257.

11. Abrutyn E, Mossey J, Berlin JA, et al. Does asymptomatic bacteriuria predict mortality and does antimicrobial treatment reduce mortality in elderly ambulatory women? Ann Intern Med 1994; 120:827-833.

12. Filice GA, Drekonja DM, Thurn JR, Hamann GM, Masoud BT, Johnson JR. Diagnostic Errors that Lead to Inappropriate Antimicrobial Use. Infect Control Hosp Epidemiol 2015; 36:949-956.

13. Rodhe N, Löfgren S, Matussek A, et al. Asymptomatic bacteriuria in the elderly: high prevalence and high turnover of strains. Scand $\mathrm{J}$ Infect Dis 2008; 40:804-810.

14. Kapasi AJ, Dittrich S, González IJ, Rodwell TC. Host Biomarkers for Distinguishing Bacterial from Non-Bacterial Causes of Acute Febrile Illness: A Comprehensive Review. PLoS ONE 2016; 11:e0160278.

15. Schuetz P, Albrich W, Mueller B. Procalcitonin for diagnosis of infection and guide to antibiotic decisions: past, present and future. BMC Med 2011; 9:107.
16. Sager R, Kutz A, Mueller B, Schuetz P. Procalcitonin-guided diagnosis and antibiotic stewardship revisited. BMC Med 2017; 15:15.

17. Drozdov D, Schwarz S, Kutz A, et al. Procalcitonin and pyuria-based algorithm reduces antibiotic use in urinary tract infections: a randomized controlled trial. BMC Med 2015; 13:104.

18. Xu R-Y, Liu H-W, Liu J-L, Dong J-H. Procalcitonin and C-reactive protein in urinary tract infection diagnosis. BMC Urol. 2014; 14:45.

19. Levine AR, Tran M, Shepherd J, Naut E. Utility of initial procalcitonin values to predict urinary tract infection. Am J Emerg Med 2018; 36:1993-1997.

20. Shaikh KJ, Osio VA, Leeflang MM, Shaikh N. Procalcitonin, C-reactive protein, and erythrocyte sedimentation rate for the diagnosis of acute pyelonephritis in children. Cochrane Database Syst Rev 2020; 9:CD009185.

21. Sugimoto K, Shimizu N, Matsumura N, et al. Procalcitonin as a useful marker to decide upon intervention for urinary tract infection. Infect Drug Resist 2013; 6:83-86.

22. Lai C-C, Chen S-Y, Wang C-Y, et al. Diagnostic value of procalcitonin for bacterial infection in elderly patients in the emergency department. J Am Geriatr Soc 2010; 58:518-522.

23. Dwolatzky T, Olshtain-Pops K, Yinnon AM, et al. Procalcitonin in the elderly: normal plasma concentrations and response to bacterial infections. Eur J Clin Microbiol Infect Dis 2005; 24:763-765.

24. Kuil SD, Hidad S, Fischer JC, et al. Sensitivity of C-reactive protein and procalcitonin measured by Point-of-Care tests to diagnose urinary tract infections in nursing home residents: a cross-sectional study. Clin. Infect. Dis. 2020; [Online ahead of print].

25. Nicolle LE. Uncomplicated urinary tract infection in adults including uncomplicated pyelonephritis. Urol Clin North Am 2008;35: 1-12.

26. Colgan R, Williams M. Diagnosis and treatment of acute uncomplicated cystitis. Am Fam Physician 2011;84:771-6.

27. Harris PA, Taylor R, Thielke R, Payne J, Gonzalez N, Conde JG. Research electronic data capture (REDCap)--a metadata-driven methodology and workflow process for providing translational research informatics support. J Biomed Inform 2009; 42:377-381.

28. Harris PA, Taylor R, Minor BL, et al. The REDCap consortium: Building an international community of software platform partners. $J$ Biomed Inform 2019; 95:103208.

29. Schisterman EF, Perkins NJ, Liu A, Bondell H. Optimal cut-point and its corresponding Youden Index to discriminate individuals using pooled blood samples. Epidemiology 2005; 16:73-81.

30. Arinzon Z, Shabat S, Peisakh A, Berner Y. Clinical presentation of urinary tract infection (UTI) differs with aging in women. Arch Gerontol Geriatr 2012; 55:145-147.

31. Aspevall O, Hallander H, Gant V, Kouri T. European guidelines for urinalysis: a collaborative document produced by European clinical microbiologists and clinical chemists under ECLM in collaboration with ESCMID. Clin Microbiol Infect 2001;7:173-8.

32. Hanley JA, McNeil BJ. The meaning and use of the area under a receiver operating characteristic (ROC) curve. Radiology 1982; 143:29-36.

33. Krzanowski W, Hand D. ROC curves for continuous data. Chapman and Hall/CRC, 2009.

34. Sultana RV, Zalstein S, Cameron P, Campbell D. Dipstick urinalysis and the accuracy of the clinical diagnosis of urinary tract infection. J Emerg Med 2001; 20:13-19.

35. Rothe K, Spinner CD, Waschulzik B, Janke C, Schneider J, Schneider H, Braitsch K, Smith C, Schmid RM, Busch DH, Katchanov J. A diagnostic algorithm for detection of urinary tract infections in hospitalized patients with bacteriuria: The "Triple F" approach supported by Procalcitonin and paired blood and urine cultures. PLoS One 2020;15:e0240981.

36. Meister L, Morley EJ, Scheer D, Sinert R. History and physical examination plus laboratory testing for the diagnosis of adult female urinary tract infection. Acad Emerg Med 2013;20:631-45.

Publisher's Note: Springer Nature remains neutral with regard to jurisdictional claims in published maps and institutional affiliations. 